\title{
Agent-based Holonic Production Control
}

\author{
Paulo Leitão \\ Polytechnic Institute of Bragança \\ Quinta $S^{\text {ta }}$ Apolónia, Apartado 134 \\ P-5301-857 Bragança, Portugal \\ pleitao@ipb.pt
}

\author{
Francisco Restivo \\ Faculty of Engineering, University of Porto \\ Rua Dr. Roberto Frias \\ P-4200-465 Porto, Portugal \\ fjr@fe.up.pt
}

\begin{abstract}
The manufacturing system environment is typically a complex system, involving many variables and constraints, being in certain cases a chaotic system. The introduction of new paradigms to face globalisation, distribution of activities and customer satisfaction requirements, increases the problem complexity. The new manufacturing control approaches should support the agile adaptation to volatile technological and economical environments and should react dynamically and quickly to disturbances. This paper intends to introduce an agent-based approach to the manufacturing problem, that uses holonic concepts, is focused on distributed manufacturing shop floor control for discrete batch production, considers the optimisation of set-up and maintenance operations, and develops mechanisms for agile and fast reaction to disturbances without compromising the global production optimisation.
\end{abstract}

\section{Introduction}

The production domain, where the manufacturing systems belong, is and will be in the future one of the main wealth generators, assuming a crucial importance the analysis, design and implementation of new and innovative manufacturing control systems, in order to establish a solid base for the economic growth. Manufacturing systems involve activities related to the production of several different products, each of them with many different customisations.

On the other hand, in the last decades it has been noticed a significant and gradual change in the manufacturing environment, that is moving from a local economy towards a global economy, with markets demanding mass customisation and imposing new requirements, such as product diversity and shorter product life-cycle, leading the enterprise to adapt to those new requirements, to avoid the risk to loose competitiveness.

The achievement of efficient manufacturing planning and control systems that minimize the non-productive times is crucial to optimise the global production. However, the occurrence of disturbances in the manufacturing system (both machine failures, layout reconfiguration and product changes) leads to deviations from the initial plans and causes delays and no-operative situations, justifying the need of mechanisms that react to disturbances in order to minimize their effects.

The holonic manufacturing concept and the agentbased manufacturing approaches that have been introduced in the manufacturing domain by several research teams, such as referred in $[1,2,3,4,5]$ and others compiled in [6], allow a new approach to the manufacturing problem, through the concepts of modularity, decentralization, autonomy, re-use of software components, etc.

In this paper it is introduced a new approach to distributed manufacturing systems that aims to develop mechanisms to support the fast and agile reaction to disturbances, without compromising the global production optimisation. The approach is based in an autonomous and cooperative agent platform, and in the distributed control and scheduling concepts, that support mechanisms to adapt quickly to the disturbances.

\section{Manufacturing Problem Formulation}

The manufacturing problem can be divided mainly into the components taxonomy and the problem formalisation. The product, order and resource taxonomies contribute to the definition of a manufacturing ontology that supports the easy development of agent-based manufacturing control applications. The problem formulation describes the goals, constraints and behaviour of the manufacturing 
environment, at the planning, scheduling and control levels.

\subsection{Product, Order and Resource Taxonomy}

The manufacturing enterprises produce products that are offered to the market. The products are described by the product model, which contains all technical data and the constitution of a product, and by the process model, which defines how to produce the product. A part is generally a material item that is used as a component and is not an assembly, subassembly, blend intermediate, etc. The process plan defines a list of operations and related information, sequence, estimated processing time and requirements that are necessary to produce the part. An operation is a job to be executed in one location that involves one of the following main functions: processing, assembly, storage, transportation, manipulation, maintenance or inspection. Each operation has aggregated a set of services to perform the operation.

A customer interacts with a company to order one of the available products or a new specified product. This order, known as customer order, involves the reference to a product, a quantity, a deliver date and a price. Additionally, based in market analysis and past information, it is necessary to create forecast orders to anticipate the market demands. The manufacturing planning convert the customer and forecast orders into manufacturing orders, aggregating if possible several customer orders into a manufacturing order, to obtain volume and transport advantages or discounts. The manufacturing orders must specify a quantity, a delivery date and a cost. A manufacturing order is indexed to a product object and comprises a list of work orders, according with the product related process plan. A work order is a job that should be executed by a resource.

Resource is an entity that can execute a certain range of jobs, when it is available, as long as its capacity is not exceeded. A resource is defined by its functional attributes (work volume, cutting speed, spindle speed, types of allowed material, list of tools and grippers, etc). The availability of a resource is represented by an agenda that indicates the list of orders allocated to the resource over the time and the free time slots. The agenda comprises time slots where the agent is: free, allocated to execute orders, temporarily out of service (for example for maintenance) and out of service.

\subsection{Manufacturing Control System Problem}

The main functions required by a manufacturing control system are process planning, resource allocation planning, resource allocation plan execution and pathological state handling.

The execution of a product involves the execution of several parts, according to a precedence diagram, defined in the process plan for the product. The precedence is a constraint that increases the problem complexity. At the process planning level, the manufacturing orders are launched to the shop floor, associated to a process plan that defines the required sequence of operations to be executed and the required machine type for each operation. Based on the available resources in the system, it is possible to create alternative process sequences, each one indicating the exact resource to execute each operation.

The resource allocation planning schedules the necessary operations to produce the parts, including processing, transport, maintenance and set-up operations, taking into account the process plans, the constraints and resources capacity, in order to produce the products, minimizing the costs and increasing the productivity, and organizing the production unit to react to any modification in demand or machine failure.

The resource allocation plan execution functions deals with the operational implementation of the schedule into the factory through the dispatching of the scheduled orders to the manufacturing process, and with the production progress monitoring.

The reaction to disturbances is initially taken by the execution plan level, and may imply the need of rescheduling of the operations with the aim of minimizing the effects of the disturbance.

\section{The ADACOR Approach}

The ADACOR [7] architecture is focused on distributed manufacturing shop floor control, for the job shop production type, considering the optimisation of setup and maintenance operations, and developing mechanisms for the agile and fast reaction to disturbances, using an adaptive control approach.

\subsection{Holonic based Architecture}

The ADACOR uses an holonic approach, based on a set of autonomous and cooperative holons, where each holon represents a manufacturing entity, such as a machine-tool, a robot, and a manufacturing order. Each holon may perform several roles, depending of the actual scenario, and may be member of several groups, performing one role in each group.

The implementation of the holons entities and its organizational behaviour is done using the agent 
technology. In terms of origin, the agents have their roots in the computer science and the holons in the computer integrated manufacturing domain, focusing the problems associated to the flexible manufacturing systems. But in our view the concepts of holon and agent are quite different, in spite of the similarities.

- In conceptual terms, the holon is a concept and an agent is both a concept and a technology, being possible to implement the holon concept and a holonic manufacturing systems using agent technology.

- The agents normally represent software components and the focus is not the integration of physical resources. The holon concept supports the integration of physical resources, based on the feature that a holon comprises logical and physical components.

\subsection{Internal Architecture of an ADACOR Holon}

As the ADACOR architecture is based in a set of autonomous and co-operative holons, it is crucial the specification of an architecture for a generic ADACOR holon. The generic holon in ADACOR approach comprises the physical resource, capable to perform manufacturing tasks (if applicable), and the Logical Control Device (LCD) implemented using agent technology. The internal architecture for the LCD device, as shown in Figure 1, comprises a local knowledge base and three main components [7]: decision, communication and physical interface component.

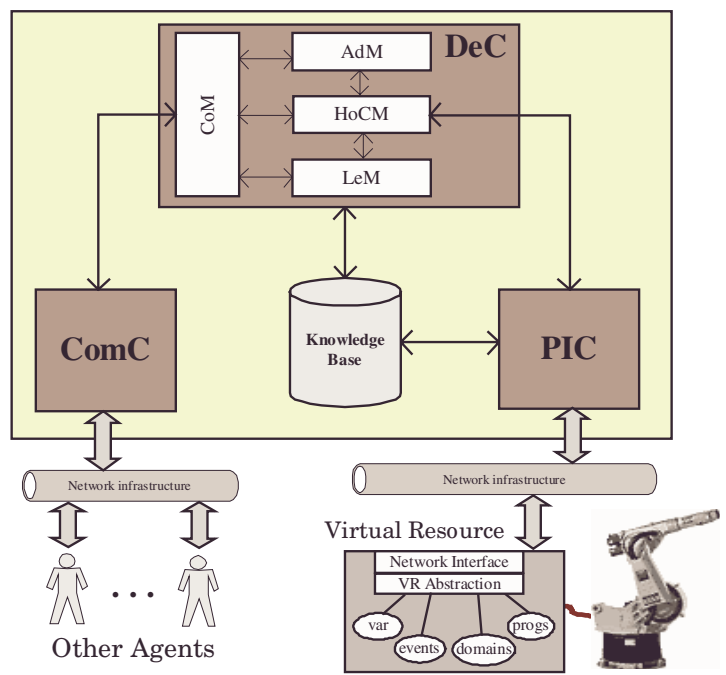

Figure 1 - Internal Architecture of an ADACOR Holon

The decision component (DeC), developed in a rulebased programming language, controls the activities of the agent based in a behaviour engine, and comprises the holon control (HoCM), cooperation (CoM), adaptation (AdM) and learning (LeM) modules. The HoCM module intents to control the behaviour of the holon, performing the planning, resource allocation, dispatching, monitoring and reaction to disturbances functionalities. The cooperation module manages the interaction and conversation with the other holons, supporting several simple interaction protocols. The AdM module allows the dynamic re-organisation of the holons into different organisational structures, in order to adapt to the organisational structures changes and in reaction to the disturbances. The LeM module handles with the environment volatility due to disturbances and improve the holon performance and abilities.

The communication component (ComC) deals with the interaction with other holons, making transparent the data exchange by using appropriate ontologies and the FIPA-ACL as communication language.

A crucial point when agentifying manufacturing components is the connection between the agent and the manufacturing resource. As the local resource controllers have close architectures it is necessary to develop wrappers to hide the details of each resource controller and supplies primitives that represent the functionality of the physical manufacturing resource. The physical interface component (PIC) provides mechanisms that makes transparent the access to manufacturing resources, supported by the Virtual Resource concept inspired in Virtual Manufacturing Device concept from MMS (Manufacturing Message Specification) protocol [8], that provides a standard access using a set of services, grouped in re-use libraries, and invoked remotely by the holon to access to the manufacturing resource.

The local knowledge base stores all knowledge about the behaviour of the holon and the community where the agent belongs, such as goals, constraints, skills, behaviour rules, plans, organisational structures, and dynamical information.

\subsection{ADACOR Entities}

The ADACOR approach is based on several holon types: the operational, supervisor, task and product holons [7].

Each product is represented by a product holon that contains all knowledge related to the product and is responsible for the process planning. Based in the knowledge related to the operations routing and in the available resources in the system, the product holon elaborates several alternative plans for the execution of the product, indicating for each operation a concrete resource to execute it.

Each available manufacturing order is represented by a task holon, which is responsible for the control and 
supervision of the execution of a manufacturing order and contains the dynamic information about the manufacturing order.

The operational holons represent the physical resources, such as operators, robots and NC machines, managing its behaviour according the resource goals, constraints and skills, and optimising its schedule agenda.

The supervisor holon has the objective of coordinating several operational and supervisor holons, introducing coordination features and global optimisation.

\subsection{Distributed Scheduling}

The scheduling problems are complex combinational problems, becoming harder when the number of machines and operations increases. Since the experience show that in manufacturing environments it is not important to have the best solution but a satisfactory solution, there are scheduling heuristics, such as EDD (Earliest Due Date), which allow a good approach to the ideal solution, in a shorter period of time, but cannot be applied for the global problem of several machines and several resources.

In the ADACOR approach a distributed and dynamic scheduling that supports efficiently the agile and fast reaction to the disturbances is introduced. The distributed scheduling mechanism uses a multi-round Contract Net protocol with some extensions, to support multiple iterations and bid partial quantities. In this approach each operational holon has a local scheduling engine and is responsible for its own schedule, build from the local knowledge and goals, in order to optimise the global performance. The global scheduling is achieved by the interaction between the operational and the task holons.

\section{Adaptive Control Concept}

The ADACOR architecture supports different control approaches, such as the hierarchical, federation and heterarchical, but in order to increase the agility to unexpected disturbances without compromising the global production optimisation, it is necessary to evolve to a new control approach that combines those requirements. The proposed control approach is achieved by splitting the control into alternative states: steady state and transient state.

\subsection{Steady State}

In the steady state, the holons in the system are organised in a federation control approach, using supervisor holons to coordinate a set of other supervisor and/or operational holons. In this federation structure, the supervisor holons represent cells and/or the shop floor, and have coordination functions through the elaboration of optimised schedule plans for the supervisor and/or operational holons in its coordination domain.

The task holons interact with the supervisor holons, which will interact with other supervisor and operational holons, hierarchically in lower level, in order to allocate the manufacturing orders. The supervisor holon elaborates optimised schedule for its coordinated resources, and dispatches it to the operational and supervisor holon that have enough autonomy to accept or reject the proposed schedule.

\subsection{Transient State}

The need to react rapidly to a disturbance that requires the update to the original plans may not be satisfied by the steady state. In this case the system re-organise itself for a short period of time, with each operational holon adjusting its autonomy factor, applying a general mechanism that comprises four important steps, as shown in Figure 2.

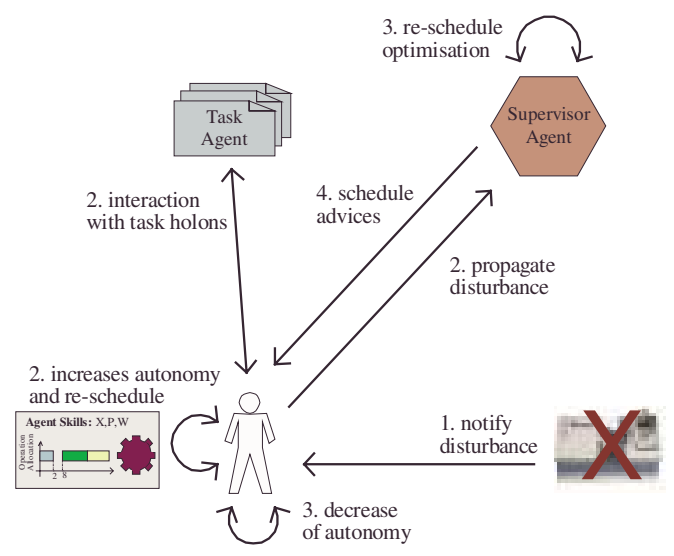

Figure 2 - Transient State Mechanism

Initially, the operational holon increases slightly the autonomy factor and tries to recover locally the failure, re-scheduling its operations, in order to minimize the deviation to the original plan. In case of success, the operational holon reduces again the autonomy factor and the disturbance is recovered. If the operational holon cannot recover from the failure or it cannot fulfil the due date of an operation, the operational holon increases the autonomy factor, according with the type of disturbance, and disseminates the need for a re-organisation into a heterarchical organisational structure to other holons. In parallel the operational holon re-schedule the operations, interacting with the task holons if necessary to notify the disturbance. 
After the recovery of disturbance the operational holon should synchronize its schedule with the optimised schedule, notifying the supervisor holon about the new schedule, and reducing again its autonomy factor. Since the autonomy factor is reduced the dissemination of disturbance is finished and the other holons doesn't sense anymore the occurrence of disturbance, reducing as well its autonomy factor, returning the system to the previous structure (hierarchical or federated form).

\section{Limitations and Advantages}

The introduction of holonic concepts and multi-agent technology in manufacturing environment brings a set of significant advantages.

The autonomy and cooperation features of holons support the distribution of functions, knowledge and skills, allowing the modular design of the control application, and the introduction of decentralized control, which increases the agility to react to unexpected disturbances. The adaptive control mechanism allows the control system to adapt quickly to disturbances, maintaining the global production optimization.

The re-configuration during the application life cycle, for example the addition of new components, a frequent scenario in manufacturing systems, is possible without the need of re-design, re-programming and reinitialisation of other components. Since the holonic control application is designed as a set of autonomous holons, each one designed to adapt its behaviour in order to achieve its own goals, the re-configuration of the structure is easier than in the traditional approaches, because the holons have independent behaviour and autonomous decisions.

The current implementation of ADACOR architecture shows some limitations that may be overcome in the future. First of all, the approach is focused in the job shop production type and the set-up operations and tool management are not considered. The autonomy factor concept needs further development. At the moment, fuzzy logic and neural networks are being considered for the implementation of the autonomy factor.

\section{Conclusions}

The manufacturing environment is typically a complex and chaotic system, involving many constraints and aiming to achieve an optimised resource allocation in order to improve the manufacturing productivity. To face the manufacturing constraints it is necessary to have efficient and innovative manufacturing control systems that adapt quickly to disturbances, increasing the enterprise productivity. This paper intends to present an holonic approach to the manufacturing problem, focused on distributed manufacturing shop floor control, which presents mechanisms for agile and fast reaction to disturbances implying a dynamic, hybrid and adaptive control. This approach uses a set of holons to support the control distribution, being each holon capable to take decisions and to organise in different structures in order to optimise its goals, allowing an easier development and understating of the manufacturing control problem through the division of the complex problem into small and simples problems. The adaptive control concept is introduced, allowing to increase the capability to an agile adaptation to disturbances.

\section{References}

[1] Parunak, H. Van Dyke, A. Baker and S. Clark (1998) The AARIA agent architecture: from manufacturing requirements to agent-based system design, Workshop on Agent-based Manufacturing, ICAA'98, Minneapolis.

[2] Van Brussel, H., J. Wyns, P. Valckenaers, L. Bongaerts and P. Peeters (1998), Reference Architecture for Holonic Manufacturing Systems: PROSA, Computers In Industry, vol. 37, pp. $255-274$

[3] Shen, W., D. Xue and D. Norrie (1998), An Agent-Based Manufacturing Enterprise Infrastructure for Distributed Integrated Intelligent Manufacturing Systems, in proceedings of the Third International Conference on the Practical Application of Intelligent Agents and Multi-Agents, London, UK, March, 23-25.

[4] Fisher, K. (1999), Agent-Based Design of Holonic Manufacturing Systems, Journal of Robotics and Autonomous Systems, Elsevier Science B.V., vol.27, pp 3-13.

[5] Brennan, R., S. Balasubramanian, and D. Norrie (1997), A dynamic control architecture for metamorphic control of advanced manufacturing systems, in the Proceedings of the International Symposium on Intelligent Systems and Advanced Manufacturing, ed. by B. Gopaladrishnan, S. Murugesan, O. Struger, and G. Zeichen, pp. 213-223.

[6] Journal of Applied Systems Studies, Special Issue on Industrial Applications of Multi-Agent and Holonic Systems, 2(1), 2001.

[7] Leitão, P. and F. Restivo (2001), An Agile and Cooperative Architecture for Distributed Manufacturing Systems, in Proceedings of the IASTED International Conference Robotics and Manufacturing, 21-24 May, Cancun, Mexico, pp. 188-193.

[8] ISO/IEC 9506-1 (1992), Industrial Automation Systems Manufacturing Message Specification, Part 1 - Service Definition. 